University of Nebraska - Lincoln

DigitalCommons@University of Nebraska - Lincoln

June 1990

\title{
Photodarkening profiles and kinetics in chalcogenide glasses
}

Stephen Ducharme

University of Nebraska - Lincoln, sducharme1@unl.edu

J. Hautala

Department of Physics, University of Utah - Salt Lake City

P.C. Taylor

Department of Physics, University of Utah - Salt Lake City

Follow this and additional works at: https://digitalcommons.unl.edu/physicsducharme

Part of the Physics Commons

Ducharme, Stephen; Hautala, J.; and Taylor, P.C., "Photodarkening profiles and kinetics in chalcogenide glasses" (1990). Stephen Ducharme Publications. 23.

https://digitalcommons.unl.edu/physicsducharme/23

This Article is brought to you for free and open access by the Research Papers in Physics and Astronomy at DigitalCommons@University of Nebraska - Lincoln. It has been accepted for inclusion in Stephen Ducharme Publications by an authorized administrator of DigitalCommons@University of Nebraska - Lincoln. 


\title{
Photodarkening profiles and kinetics in chalcogenide glasses
}

\author{
Stephen Ducharme, ${ }^{*}$ J. Hautala, and P. C. Taylor \\ Department of Physics, University of Utah, Salt Lake City, Utah 84112
}

(Received 4 December 1989)

\begin{abstract}
The spatial and temporal evolution of photodarkening in chalcogenide glasses is simulated by a simple kinetic model and tested against experiments. In the model, photodarkening, a gradual decrease of the energy of the absorption edge caused by above-band-gap illumination, is the additional absorption at defects created by the absorbed light. The defect-creation rate is proportional to the rate at which photons are absorbed either directly at the defect site or, alternatively, at a neighboring site. The depletion of the incident light within the sample is included and the resulting equations cast into a universal form that is applicable to realistic situations. The equations contain as adjustable parameters only the time and length scales. Measurements of the spatial and temporal evolution of photodarkening in bulk glasses of arsenic sulfide $\left(\mathrm{As}_{2} \mathrm{~S}_{3}\right)$ illuminated with green (5145$\AA$ A) light at intensities from 0.1 to $7 \mathrm{~W} / \mathrm{cm}^{2}$ for up to four days are reported. Melt-quenched samples as thin as $2.4 \mu \mathrm{m}$ have been employed. These results quantitatively fit one form of the kinetic model. The experiments also determine the approximate low-temperature values of the saturated absorption coefficient $\left(\sim 4000 \mathrm{~cm}^{-1}\right)$, the characteristic profile depth $(\sim 2 \mu \mathrm{m})$, and the initial photodarkening efficiency $(44 \mathrm{~cm} / \mathrm{J})$ in $\mathrm{As}_{2} \mathrm{~S}_{3}$ glass.
\end{abstract}

\section{INTRODUCTION}

Certain semiconducting glasses whose common feature is the presence of a chalcogenide atom $(\mathrm{O}, \mathrm{S}, \mathrm{Se}$, or Te) exhibit a metastable shift of the optical-absorption edge to longer wavelengths (e.g., a decrease or an apparent decrease of the band gap) when the glass is illuminated with strongly absorbed light. This metastable shift of the absorption edge, and consequent increase of absorption at fixed wavelengths is commonly called" "photodarkening." Photodarkening has been observed in glasses of $\mathrm{As}_{2} \mathrm{~S}_{3},{ }^{2} \mathrm{As}_{2} \mathrm{Se}_{3},{ }^{2} \mathrm{GeSe}_{2},{ }^{3} \mathrm{GeS}_{2},{ }^{3} \mathrm{Se},{ }^{4}$ and $\mathrm{As}_{2} \mathrm{O}_{3},{ }^{5}$ as well as in many other binary, ternary, and quaternary combinations, which all contain twofold-coordinated chalcogen atoms. The effect is not observed in amorphous $\mathrm{As},{ }^{6} \mathrm{Si}, \mathrm{Se}$, or $\mathrm{Sn}$ which do not contain chalcogen atoms. Photodarkening effects are strongest at low temperature, though they are often observable even at room temperature. Photodarkening can be removed by annealing the sample just below the glass transition temperature. Shifts of the absorption edge up to $0.15 \mathrm{eV}$ at $80 \mathrm{~K}$ have been observed $^{7}$ in $\mathrm{As}_{2} \mathrm{~S}_{3}$ films which have an optical band gap of $\sim 2.4 \mathrm{eV}$ at $300 \mathrm{~K}$.

Photodarkening is generally accompanied by other changes caused by above-band-gap illumination at low temperatures: the appearance of paramagnetic centers ${ }^{8}$ (spins) with $g \simeq 2$, increases in midgap absorption, ${ }^{9}$ a decrease in material density, ${ }^{10}$ and fatigue of the photoluminescence. ${ }^{11}$ But both the light-induced spins and the increased midgap absorption have been shown to be caused by defects only indirectly related to those producing photodarkening: (1) the spins and increased midgap absorption have been induced by below-gap light which produced no measurable photodarkening; ${ }^{12}$ (2) the spins and midgap absorption induced while photodarkening with above-band-gap illumination can be fully annealed out at temperatures which only partially anneal the photodarkening; ${ }^{13,14}$ (3) copper alloyed $\mathrm{As}_{2} \mathrm{~S}_{3}$ and $\mathrm{As}_{2} \mathrm{Se}_{3}$ glasses exhibit light-induced spins and midgap absorption but no photodarkening is induced by above-band-gap illumination. ${ }^{15}$ Though photodarkening has sometimes been observed separately from light-induced spins and midgap absorption, the creation of these three effects under some circumstances may not be independent. The remaining discussion will primarily concern the behavior of the photodarkening upon illumination with near and above-band-gap light.

Photodarkening behavior in chalcogenide glasses is qualitatively similar in vapor-deposited thin films and in melt-quenched bulk samples but there are important physical and practical differences between film and bulk samples. Films, unlike bulk glasses, often contain significant amounts of off-stoichiometric material (such as $\mathrm{As}_{4} \mathrm{~S}_{4}$ in $\mathrm{As}_{2} \mathrm{~S}_{3}$ films) even after annealing. The films also exhibit irreversible structural changes under illumination, ${ }^{16}$ changes not observed in their bulk counterparts. But it is easier to measure large absorption and photodarkening, as well as volume changes in films than it is in much thicker bulk samples. In a bulk sample, the absorption of the inducing light in the first few micrometers of the sample makes it difficult to photodarken a sample uniformly and completely when the sample thickness is greater than a few $\mu \mathrm{m}$. We have succeeded in observing saturated photodarkening by polishing bulk samples to thicknesses of a few micrometers.

The next section describes two kinetic models of defect creation during photodarkening. The coupled differential equations in time and (one-dimensional) space are reduced to unitless form and numerically integrated to yield the evolution of the photodarkening profiles in time. 
Defect diffusion is neglected in both models. Although diffusion lengths are not known, they are probably small ${ }^{17}$ in comparison to experimentally employed sample thicknesses which are greater than $\sim 1 \mu \mathrm{m}$. Section III describes the sample preparation and experimental techniques used to measure both the spatially averaged time evolution of induced absorption and also the actual profiles in a number of samples of $\mathrm{As}_{2} \mathrm{~S}_{3}$ glass with thicknesses ranging from $2.4 \mu \mathrm{m}$ to $6 \mathrm{~mm}$. In Sec. IV, the experimental data are compared to the model calculations and important physical parameters are extracted.

\section{KINETIC MODEL OF PHOTODARKENING}

Here we derive the equations for two similar kinetic models for the temporal and spatial evolution of photodarkening in thick samples. We assume that metastable structural defects are produced one at a time by light quanta either directly or indirectly and that these defects do not diffuse. Tanaka and Ohtsuka ${ }^{18}$ have proposed a phenomenological model of photodarkening in which the increase in absorption at a fixed wavelength is proportional to the intensity and a factor ensuring saturation at some maximum absorption. They included the fact that after photodarkening the absorption depends upon position in the sample and then solved the resulting coupled differential equations numerically. They obtained good agreement between their solutions and experiments on thin films of $\mathrm{As}_{2} \mathrm{~S}_{3}$ glass. This kinetic model was further generalized by Liu and Taylor ${ }^{19}$ who assumed that the rate of increase of the absorption is proportional to a general, but unspecified, function of the absorption itself and to a power of the intensity. In addition, Liu and Taylor considered two specific cases, a form identical to that of Tanaka and Ohtsuka ${ }^{18}$ and a form identical to the indirect defect-creation process described below. In this section we derive the rate equations for defect production under illumination by considering individual single photon events. We equate the defect density to increased absorption, include explicitly the fact that the absorption coefficient is a function of position in the sample, and transform the resulting equation to a simple unitless and scale invariant form. This equation is then solved numerically for the temporal and spatial dependence of photodarkening from first illumination to saturation for samples of various thicknesses. Two forms for the initial defect-creation rate are considered, a direct process and an indirect process.

The creation of metastable defects can be represented by a configurational coordinate diagram such as that shown schematically in Fig. 1. (This diagram has also been employed by Tanaka. ${ }^{7}$ ) The ground and first excited state energies of an electron vary with a continuous distortion of the atomic configuration. Although the diagram is schematic, the features essential to photodarkening are present, in particular the metastability of the photodarkened state in a distorted configuration.

The direct process begins with electronic excitation by a photon absorbed at a nonphotodarkened site (step 1 in Fig. 1). These sites may be preexisting defects or the sites may be an ordinary atom or bond in the "ideal" glass.

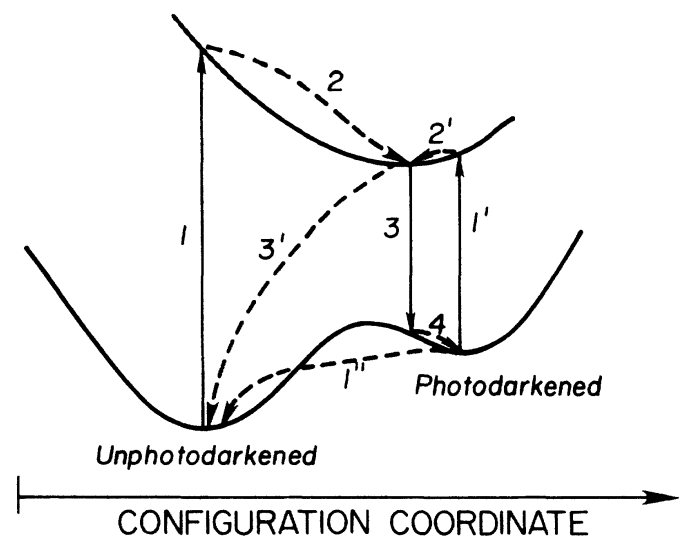

FIG. 1. Configurational coordinate diagram of the variation of ground and excited state energy levels of a bonding electron as the surrounding lattice is distorted. The transitions denoted by the arrows are discussed in the text.

Excitation is followed by atomic relaxation into a new local atomic configuration (step 2), by electronic relaxation (step 3), and by further relaxation into a metastable configurational defect (step 4). There is a finite probability for relaxation to the unphotodarkened minimum energy configuration at any point in the defect-creation process. One may account for the relaxation to the ground state by any of these processes before a metastable state is formed by a single total quantum efficiency for defect creation per photon absorbed. Defects may be erased by the light by electronic excitation (step 1') followed by relaxation (step $2^{\prime}$ ) and recombination (step $3^{\prime}$ ) as well as by purely thermal processes (step $\left.1^{\prime \prime}\right)$. With further assumptions to be described below, we may write a rate equation for the creation, erasure, and relaxation of the metastable defects:

$$
\begin{aligned}
\frac{\partial N_{d}(x, t)}{\partial t}= & \left(N_{s}-N_{d}\right) \sigma_{s} \phi_{s}\left(\frac{I}{h v}\right) \\
& -\sigma_{d} \phi_{d} N_{d}\left(\frac{I}{h v}\right)-\Gamma_{T} N_{d}
\end{aligned}
$$

where $N_{d}(x, t)$ is the number density of photodarkened sites, $N_{s}$ is the (constant) total number density of available sites, photodarkened and unphotodarkened; $\sigma_{s}$ and $\sigma_{d}$ are the absorption cross sections of the unphotodarkened and photodarkened sites, respectively; $\phi_{s}$ and $\phi_{d}$ are the total quantum efficiencies for the creation and erasure of photodarkened sites by photons; $I$ and $h v$ are the optical intensity and photon energy, respectively; and $\Gamma_{T}$ is the thermal defect relaxation rate. The first term in Eq. (1) is the defect-creation rate (steps $1-4$ in Fig. 1), the second term is the erasure (or bleaching) rate (steps $1^{\prime}-3^{\prime}$ ) and the final term the thermal defect relaxation rate (step $\left.1^{\prime \prime}\right)$. We will ignore the final term which is negligible compared to the first two terms at the intensities and temperatures of the experiments reported here. (See, for example, Fig. 4 of Ref. 19.) Equations (1) and (2) below 
are equivalent to those of Tanaka and Ohtsuka ${ }^{18}$ who have also examined the solutions and compared them to experiments performed on thin films of $\mathrm{As}_{2} \mathrm{Se}_{3}$.

Three assumptions are implicit in the rate Eq. (1) for the direct process. First, both creation and erasure of defects are assumed to be single photon processes. The duration of the intermediate steps $\left(2-4\right.$ or $2^{\prime}$ and $3^{\prime}$ in Fig. 1) is short compared to the mean time between incident photons at a site (steps 1 or $\left.1^{\prime}\right)$. This assumption also implies that no light is absorbed at an intermediate state (e.g., between steps 2 and 4). Second, the sites are assumed not to be correlated; that is, the creation, erasure, or relaxation of a photodarkened site is unaffected by the presence of nearby photodarkened sites. This important assumption may break down in a heavily photodarkened sample (e.g., near saturation). Third, it is assumed that the defects do not diffuse over distances as large as the absorption length of the light. Although there is some evidence ${ }^{17}$ that the diffusion lengths of defects $\mathrm{As}_{2} \mathrm{~S}_{3}$ and $\mathrm{As}_{2} \mathrm{Se}_{3}$ glasses are of the order of $0.5 \mu \mathrm{m}$, the diffusion has been associated with defects producing fatigue of the photoluminescence which we attribute to a separate set of defects than those producing photodarkening.

When the absorption coefficient at the inducing wavelength is comparable to or exceeds the inverse of the sample thickness one must included in Eq. (1) a depthdependent intensity accounting for the absorption of the light as its passes through the sample. The intensity obeys Beer's law:

$$
\frac{\partial I(x, t)}{\partial x}=-\alpha_{\text {tot }} I=-\left[\sigma_{d} N_{d}+\sigma_{s}\left(N_{s}-N_{d}\right)+\alpha_{i}\right] I
$$

where $\alpha_{i}$ is a constant background absorption independent of the absorption by the photodarkening sites. A transmission measurement of the sample will yield the spatial average of the constant background absorption and the absorption due to the photodarkening. The average change in absorption is obtained by integrating Eq. (2) to yield

$\overline{\Delta \alpha}(t) L=\ln \left(\frac{I(L, 0)}{I(L, t)}\right)=\int_{0}^{L}\left(\sigma_{d}-\sigma_{s}\right) N_{d}(x) d x$.

Equations (1) and (2) can be reduced to a convenient dimensionless form with the assignments

$$
\begin{aligned}
& F(X, \tau)=\frac{N_{d}(x, t)}{N_{s}}\left(1+\frac{\sigma_{d} \phi_{d}}{\sigma_{s} \phi_{s}}\right), P(X, \tau)=\frac{I(t, x)}{I_{0}} ; \\
& X=\alpha_{\infty} x, \quad \tau=\gamma t, \quad I_{0}=I(0,0) ; \\
& \gamma=\frac{I_{0}}{h v}\left(\sigma_{d} \phi_{d}+\sigma_{s} \phi_{s}\right) ; \\
& \alpha_{\infty}=\sigma_{d} N_{s}\left(\frac{1-\sigma_{s} / \sigma_{d}}{1+\sigma_{d} \phi_{d} / \sigma_{s} \phi_{s}}\right) ; \\
& \alpha_{0}=\alpha_{i}+\sigma_{s} N_{s} .
\end{aligned}
$$

Then the coupled equations for the dimensionless defect density (or induced absorption) $F$ and intensity $P$ are

$$
\frac{\partial F}{\partial \tau}=(1-F) P, \quad \frac{\partial P}{\partial X}=-P\left(F+\frac{\alpha_{0}}{\alpha_{\infty}}\right) .
$$

The coupled differential Eqs. (6) are identical to Eqs. (1) and (2) of Ref. 18. Equations (1) and (2) of Ref. 19 reduce to Eqs. (6) if we set $\beta=1$ and $f\left(\alpha, \alpha_{s}\right)=\left(\alpha-\alpha_{s}\right) / \alpha \alpha_{s}$.

By photodarkening we mean $\sigma_{d}>\sigma_{s}$. If we assume $\sigma_{d} \gg \sigma_{s}$ and $\sigma_{s} \phi_{s} \gg \sigma_{d} \phi_{d}$, then $F \approx N_{d} / N_{s}$ and $\gamma \approx\left(I_{0} / h v\right) \sigma_{s} \phi_{s}$, and the ultimate or saturated absorption is $\alpha_{\infty}=\Delta \alpha(\tau=\infty, 0 \leq x \leq L) \approx \sigma_{d} N_{s}$ when all sites are photodarkened. The form of Eqs. (6) shows clearly that there is only one meaningful parameter, $\alpha_{0} / \alpha_{\infty}$. In $\mathrm{As}_{2} \mathrm{~S}_{3}$ below $80 \mathrm{~K}$, as we will show below, the saturated (maximum) value of the induced absorption is approximately $4000 \mathrm{~cm}^{-1}$ while the initial absorption $\alpha_{0}$ plus any other background absorption is about $300 \mathrm{~cm}^{-1}$ at 2.4 $\mathrm{eV}$, and hence that $\alpha_{0} / \alpha_{\infty}<0.08$ which may be neglected without significantly affecting the results. The validity of this approximation was verified by numerical solution of Eq. (6) with $\alpha_{0} / \alpha_{\infty} \leq 0.1$. The two Eqs. (6) may be combined to eliminate time explicitly and to yield the single equation for the profile:

$$
\begin{aligned}
& \frac{d y(X)}{d X}=\left(1-F_{0}\right)[1-\exp (-y)]-a y, \\
& a=1+\frac{\alpha_{0}}{\alpha_{\infty}}, \quad F_{0}=F(0,0),
\end{aligned}
$$

and

$$
F(\tau, X)=1-\left(1-F_{0}\right) \exp [-y(X)]
$$

subject to the boundary condition at $X=0$ which determines the time dependence:

$$
\begin{aligned}
\frac{\partial F(\tau, X=0)}{\partial \tau} & =(1-F) \\
& \Longrightarrow F(\tau, X=0)=1-\left(1-F_{0}\right) \exp (-\tau)
\end{aligned}
$$

These results imply that

$$
y_{\tau}(X=0) \equiv \tau \text {. }
$$

Equation (7) is effectively universal because in most experimental situations $F_{0} \ll 1$ and $(a-1)=\alpha_{0} / \alpha_{\infty} \ll 1$ and therefore

$$
\frac{d y}{d X} \cong[1-\exp (-y)]-y
$$

Equation ( $\left.7^{\prime}\right)$ contains no explicit material parameters and its solutions may be applied to any experiment by adjusting the dimensionless length $\left(X_{L}=\alpha_{\infty} L\right)$ and time $(\tau=\gamma t)$ scales.

Equations (7) and ( $\left.7^{\prime}\right)$ must be solved numerically but, in the majority of cases where $\left(7^{\prime}\right)$ is applicable the calculation need only be done once. The solutions to Eq. $\left(7^{\prime}\right)$ are shown in Fig. 2 for successive times during photodarkening in samples of two different thicknesses. At early times the samples are essentially uniformly photodarkened but as the absorption increases at the front, the inducing light is attenuated near the front and, therefore, the intensity and the rate of photodarkening deep in the 

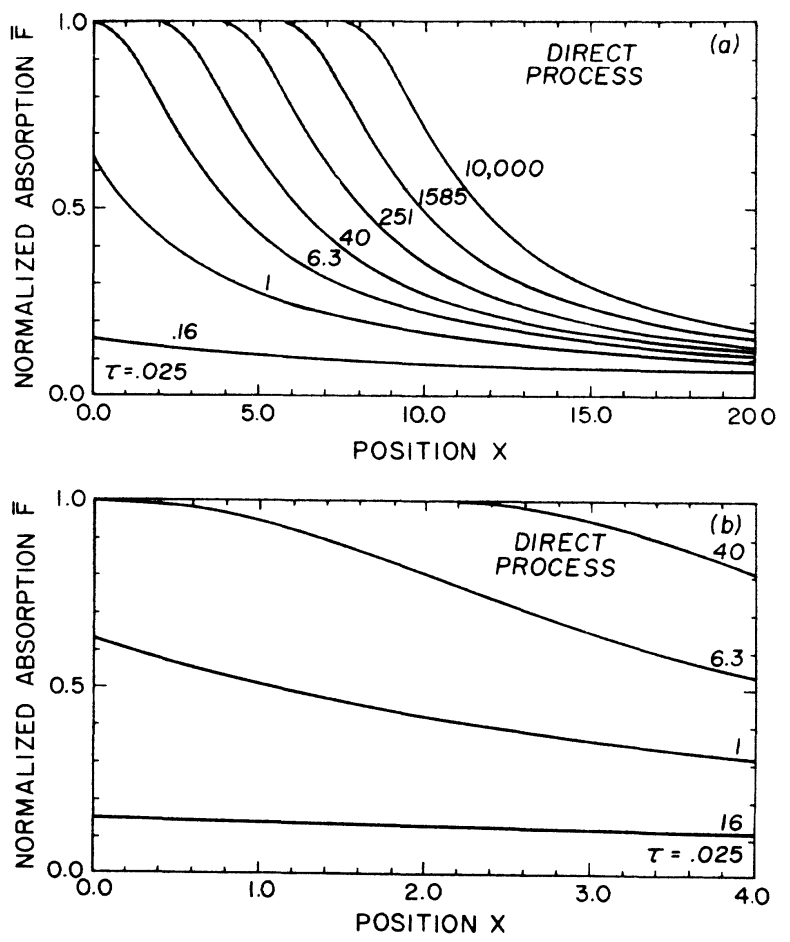

FIG. 2. Numerical solutions of Eq. (7'), the direct process, for $\alpha_{0}=0$ and (a) $\alpha_{\infty} L=20$, (b) $\alpha_{\infty} L=4$. The profiles, $F$ vs $X$, are taken at successive logarithmic time intervals as noted.

sample decrease dramatically.

The average photodarkening $\bar{F}$ which determines the fraction of light transmitted through the sample $I(L) / I(0)=\exp \left(-\alpha_{\infty} L \bar{F}\right)$ is shown for various values of $\alpha_{\infty} L$ in Fig. 3. By comparing this figure with the profiles in Fig. 2, we can see that different stages in the inducing curve $(\bar{F}$ versus $\tau)$ correspond to qualitatively different profiles. The initial stage of uniform photodarkening ( $\tau \leq 0.025$ for $\alpha_{\infty} L=20$ in Fig. 3) results in the initial flat, low valued, regions in Fig. 2(a). The sharp upturn in $F$ at $\tau \approx 0.025$ signals the second stage, the start of the rise in $F$ at the front of the sample, which culminates in saturation of the front of the sample as $\tau$ exceeds unity.

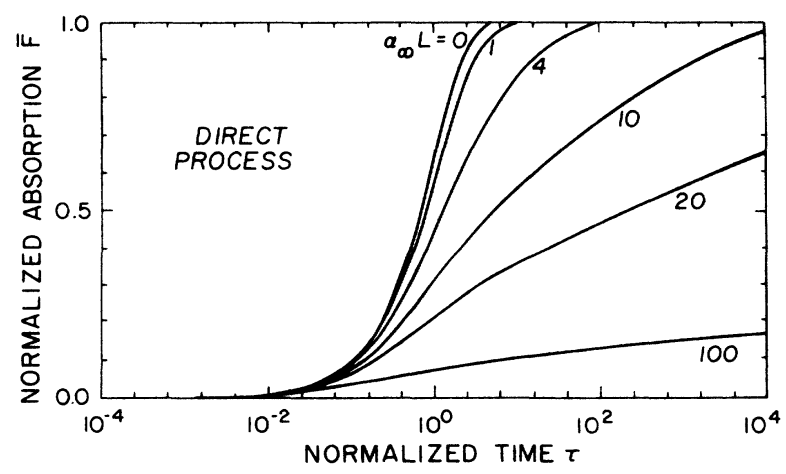

FIG. 3. The time evolution of average induced absorption coefficient $\bar{F}$ in the direct process for several values of $\alpha_{\infty} L$.
The onset of the third stage is indicated by the decrease in slope of the inducing curve, in which the position of the photodarkened "front" [defined in these calculations as the position $x$ when $F(x)=0.9$ ] advances slowly into the sample until the entire sample is photodarkened. In thick samples, this final stage is by far the longest. Figure 4(b) shows the progression of the saturation front in an infinitely thick (for practical purposes $\alpha_{\infty} L=100$ is infinitely thick) sample. From Eqs. $\left(7^{\prime}\right)$ and (4) it may be shown that the position of the photodarkening front does indeed advance proportional to $\log \tau$ for $\tau \gg>1$ and that $X_{F}(\tau)=\ln \tau-$ const, which is confirmed by the numerical results of Fig. 4(b). [Indeed, it can be shown that a necessary and sufficient condition for the $\log \tau$ advance of the photodarkening front is that the reciprocal of the righthand side of Eq. (6) is integrable with respect to $F$ when $P$ is held constant.]

The indirect process of photodarkening proposed by Liu and Taylor [Eq. (1) of Ref. 19] with $\beta=1$ and $f(\alpha)=(1-\alpha)$ encompasses a different sequence of events than the direct process. In the indirect process a photon is absorbed at an already photodarkened site with cross section $\sigma_{d}$ and the energy transferred to a nearby unphotodarkened site to photodarken it with total quantum efficiency $\phi_{s d}$. Diffusion is again neglected as each new photodarkening event takes place at a neighbor of the site
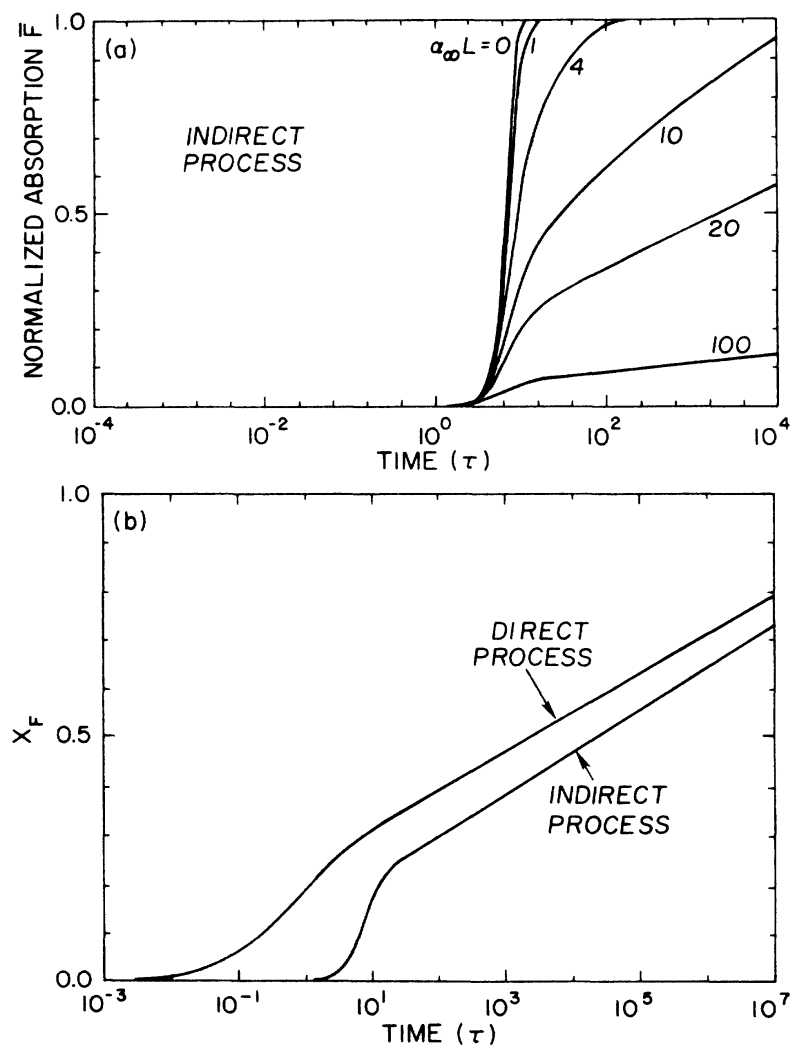

FIG. 4. (a) The time evolution of the average induced absorption coefficient $\bar{F}$ in the indirect process for several values of $\alpha_{\infty} L$ and (b) the propagation of the photodarkening "front" in an infinitely thick sample for both the direct and indirect processes. 
where the photon is absorbed and stops there. The rate equation for the photodarkening dynamics is then

$$
\begin{aligned}
\frac{\partial N_{d}(x, t)}{\partial t}= & N_{d} \sigma_{d}\left(\frac{I}{h v}\right) \phi_{s d}\left(\frac{N_{s}-N_{d}}{N_{s}}\right) \\
& -N_{d} \phi_{d} \sigma_{d}\left(\frac{I}{h v}\right)-\Gamma_{T} N_{d} .
\end{aligned}
$$

Equation (2) is then combined with Eq. (8) and converted to unitless form by using the same functional forms for $P(X, \tau), X, \tau, I_{0}$, and $\alpha_{0}$ as those shown in Eq. (4) and by

$$
\begin{aligned}
& F(X, \tau)=\left(N_{d} / N_{s}\right)\left(1-\frac{\phi_{d}}{\phi_{s d}}\right)^{-1}, \\
& \gamma=\phi_{s d} \sigma_{d} \frac{I_{0}}{h v}\left(1-\sigma_{s} / \sigma_{d}\right)\left(1-\phi_{d} / \phi_{s d}\right) ; \\
& \alpha_{\infty}=\sigma_{d} N_{s}\left(1-\phi_{d} / \phi_{s d}\right) ; \\
& \frac{\partial F}{\partial \tau}=F(1-F) P, \quad \frac{\partial P}{\partial X}=-P\left(F+\frac{\alpha_{0}}{\alpha_{\infty}}\right) ;
\end{aligned}
$$

whereas for Eqs. (5) and (6), $\alpha_{\infty} \approx \sigma_{d} N_{s}, F \approx N_{d} / N_{s}$ and $\gamma \approx\left(I_{0} / h v\right) \phi_{s d} \sigma_{d}$ for the situations in which $\sigma_{d} \gg \sigma_{s}$ and $\phi_{s d} \gg \phi_{d}$.

The first of Eqs. (10) differ from the first of Eqs. (6) only in the additional factor of $F$. We may again eliminate time explicitly to yield

$$
\frac{d y(X)}{d X}=-\ln \left(1-F_{0}+F_{0} e^{y}\right)-\delta y, \quad 0 \leq y \leq \infty
$$

with $F_{0}$ as defined in Eq. (7) and with

$$
\delta=\alpha_{0} / \alpha_{\infty}, \quad y=\ln \left(\frac{F}{F_{0}} \frac{1-F_{0}}{1-F}\right) ;
$$

and with $\delta<<F_{0}$, the "universal" profile obeys

$$
\frac{d y(X)}{d X} \cong-\ln \left(1-F_{0}+F_{0} e^{y}\right)
$$

with boundary condition $y(X=0) \equiv \tau$ as for Eq. (7).

Equation (11') is then solved as Eq. ( $\left.7^{\prime}\right)$ was with the resulting evolution in Figs. 4 and 5 for the same conditions as in Figs. 2 and 3. The same comments made for the direct process solution apply except that the absorption at the front of the sample takes a much shorter time to rise from a small value to saturation [compare Figs. 3 and 4(a)]. This difference provides our primary means of distinguishing between the direct and the indirect process experimentally.

\section{EXPERIMENTAL DETAILS}

Samples of $\mathrm{As}_{2} \mathrm{~S}_{3}$ and $\mathrm{As}_{35} \mathrm{~S}_{65}$ were produced from Cominco Electronic Materials $99.9999 \%$ pure amorphous arsenic and sulfur. The elements were sealed in 25-mm-diam quartz ampules, gradually heated (over $6 \mathrm{~h}$ ) to $540^{\circ} \mathrm{C}$, rocked for $48 \mathrm{~h}$, and then slowly cooled for 10 $\mathrm{h}$ to room temperature using a programmable controller. By gravimetric analysis the actual atomic concentrations
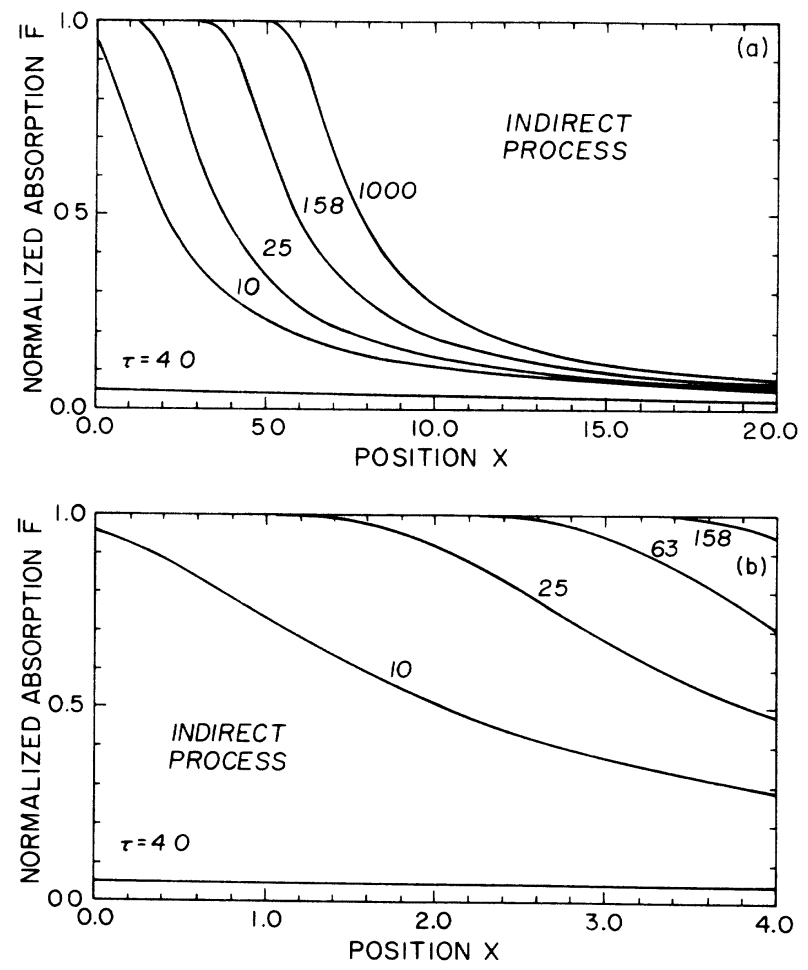

FIG. 5. Numerical solutions of Eq. (11'), the indirect process, for $\alpha_{0}=0$ and (a) $\alpha_{\infty} L=20$, (b) $\alpha_{\infty} L=4$. The profiles, $F$ vs $X$, are taken at successive logarithmic time intervals as noted.

in the glasses were found to be within \pm 0.3 at. \% of the nominal concentrations.

The transmission photodarkening experiments were arranged as in Fig. 6(a). The samples were illuminated at near normal incidence by an argon-ion laser operating at $5145 \AA$ in a TEM $_{00}$ transverse Gaussian mode. This wavelength was convenient because of the stability of the ion laser source and because this wavelength corresponds to maximum photodarkening respons $\mathrm{e}^{16}$ and to a conveniently low starting absorption coefficient at low temperatures for bulk samples ${ }^{13}$ of $300 \mathrm{~cm}^{-1}$. The transmission of the sample was probed using a monochromatic source; either tungsten lamp and monochomator, a helium neon laser, a krypton-ion laser, or a portion of the $\mathrm{Ar}^{+}$-ion laser output, and detected by a photomultiplier. The probe light was chopped at frequencies between 300 and $800 \mathrm{~Hz}$ and the photomultiplier output was connected to a lock-in amplifier referenced to the chopper frequency. The sample transmission was then measured at various wavelengths before, during, and after photodarkening, always at the same temperature as the exposure. The change in average sample absorption at each wavelength was calculated from the sample transmission using Eq. (3). The sample was immersed in liquid helium or liquid nitrogen for experiments performed at low temperatures and in a nitrogen-filled chamber for experiments at room temperature. When possible, the absorption was measured with the exciting illumination blocked to reduce effects of heating, thermal expansion, and convection in the path of the probe beam. 

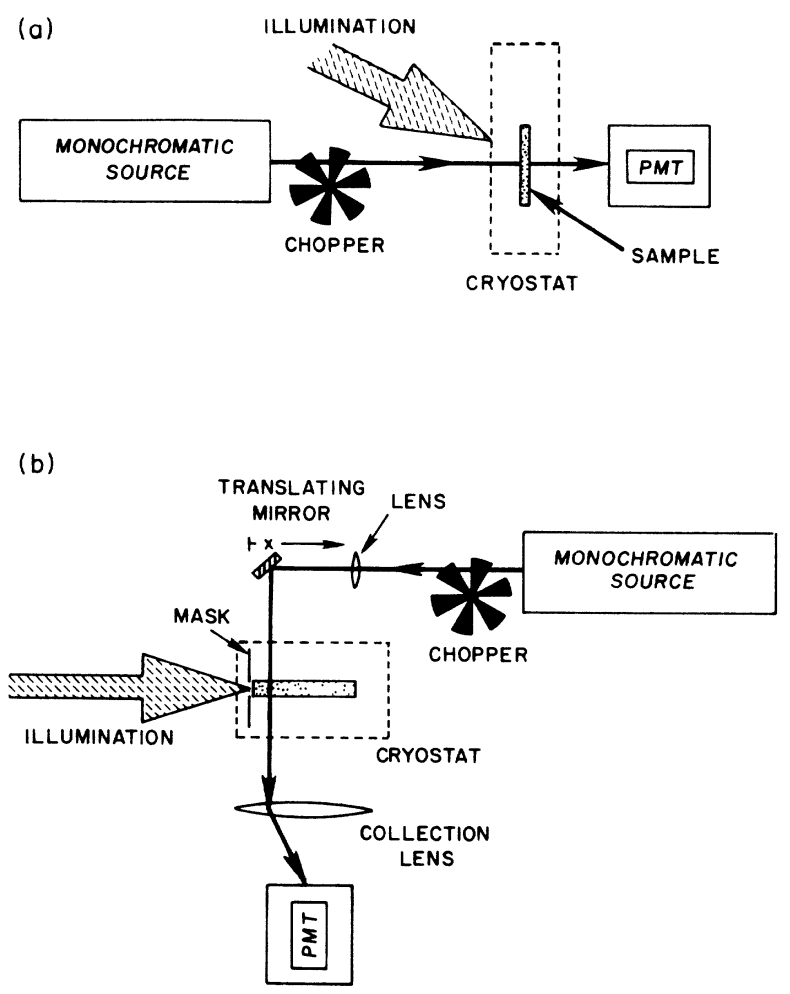

FIG. 6. (a) Collinear illumination and probe beam configuration used to measure the temporal evolution of the average sample absorption change during photodarkening. The illumination is from an argon-ion laser operating at $5145 \AA$ in a $\mathrm{TEM}_{00}$ transverse Gaussian mode. The probe beam originated from one of several sources: the same argon-ion laser, a helium neon laser, or a tungsten lamp and monochromator combination. The transmitted probe light was detected with a photomultiplier connected to a lock-in amplifier which was referenced to the source chopper frequency. (b) Perpendicular illumination and probe configuration used to measure the time and spatial evolution of the photodarkening in a thick sample. The illumination is from an argon TEM $_{00}$ transverse Gaussian mode. The probe is from a krypton-ion laser operating at $\mathbf{5 3 0 0}$ $\AA$, transmitted through a multimode fiber, spatially filtered, and focused to a $0.5-\mathrm{mm}$ spot on the sample. The transmitted beam was scanned along the sample by a micrometer-driven linear translator stage and the transmitted light collected and detected by a photomultiplier connected to a lock-in amplifier referenced to the chopper frequency.

The profile of the photodarkening deep (1-6 $\mathrm{mm})$ into thick samples was measured by scanning a probe beam oriented perpendicular to the inducing illumination as shown in Fig. 6(b). The input light was focused to a diameter less than $0.5 \mathrm{~mm}$ at the sample and moved along the sample length by the translating mirror as shown. The change in absorption $\Delta \alpha(t, x)$ was measured as for the collinear measurement [Fig. 6(a)] by taking the ratio of transmitted intensities at various positions $x$ before, during, and after illumination [Eq. (3)]. For this experiment, the illumination and probe wavelengths were 5145 and $5300 \AA$, respectively. This experiment was per- formed with the sample immersed in superfluid liquid helium at $1.8 \mathrm{~K}$. The intensity of the probe beam was low and had no measurable affect on the sample, either in producing or erasing the photodarkening.

\section{RESULTS}

The evolution of the photodarkening measured in transmission [see Fig. 6(a)] is shown in Fig. 7 for two samples of bulk quenched $\mathrm{As}_{2} \mathrm{~S}_{3}$ glass. The 17.4- and $14.7-\mu \mathrm{m}$ samples show no sign of saturation, even at the total exposure produced $\left(2.0 \mathrm{~kJ} / \mathrm{cm}^{2}\right.$ at $0.17 \mathrm{~W} / \mathrm{cm}^{2}$ and $2.3 \mathrm{~kJ} / \mathrm{cm}^{2}$ at $0.33 \mathrm{~W} / \mathrm{cm}^{2}$, respectively, where the intensities have been corrected for reflection loss assuming the sample reflectivity does not change significantly during the experiment). The inducing curves in Fig. 7 do exhibit the three stages discussed in Sec. II: initial uniform darkening $\left(<200 \mathrm{~J} / \mathrm{cm}^{2}\right)$, followed by rapid saturation of the front of the sample $\left(200-1000 \mathrm{~J} / \mathrm{cm}^{2}\right)$, then steady progression of the saturated "front" across the sample $\left(>1000 \mathrm{~J} / \mathrm{cm}^{2}\right)$. After illuminating the $14.7-\mu \mathrm{m}$ sample for the indicated time the back of the sample was illuminated and showed a similar evolution of the average absorption except that the initial, uniform darkening stage was shortened because the sample had been partially photodarkened during the first exposure. This behavior is consistent with the assumption that the illumination from the front did not darken the back of the sample significantly.

Two very thin samples were made by carefully polishing bulk glass wafers to thicknesses below $3 \mu \mathrm{m}$. These samples were exposed to illumination at $5145 \AA$ and the change in absorption at various wavelengths was monitored. The results of these exposures are displayed in Fig. 8. At $79 \mathrm{~K}$, a $2.7-\mu \mathrm{m}$-thick sample was exposed [open circles in Fig. 8(a)] to an intensity of $7.0 \mathrm{~W} / \mathrm{cm}^{2}$ for a total energy density of $147 \mathrm{~kJ} / \mathrm{cm}^{2}$. The total change in absorption reached $8500 \mathrm{~cm}^{-1}$ but when we subtract the

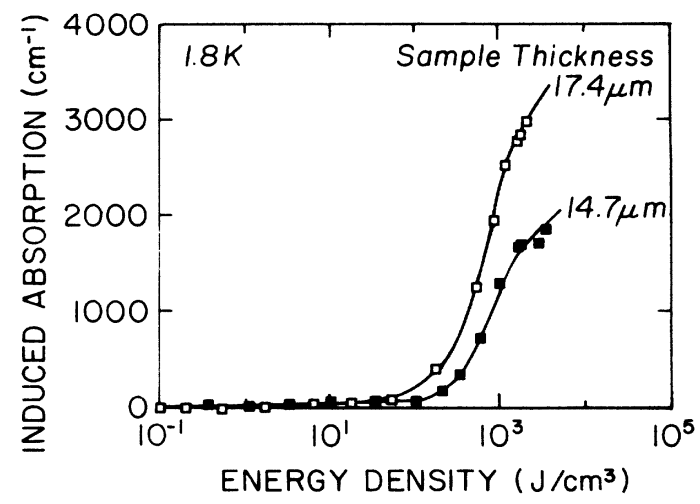

FIG. 7. Evolution of the average photodarkening $\overline{\Delta \alpha}$ in two thick samples of $\mathrm{As}_{2} \mathrm{~S}_{3}$ exposed to intense laser illumination at $5145 \AA$ while immersed in superfluid helium $(T=1.8 \mathrm{~K})$. The solution to the direct process model with $\alpha_{0}=0$ and $\alpha_{\infty}=4000$ $\mathrm{cm}^{-1}$ is plotted with each set of data (solid lines). (a) Thickness $17.4 \mu \mathrm{m}\left(\alpha_{\infty} L=7\right)$ and intensity $0.3 \mathrm{~W} / \mathrm{cm}^{2}$ (open squares). (b) Thickness $14.7 \mu \mathrm{m}$ and intensity $0.6 \mathrm{~W} / \mathrm{cm}^{2}$ (solid squares). 


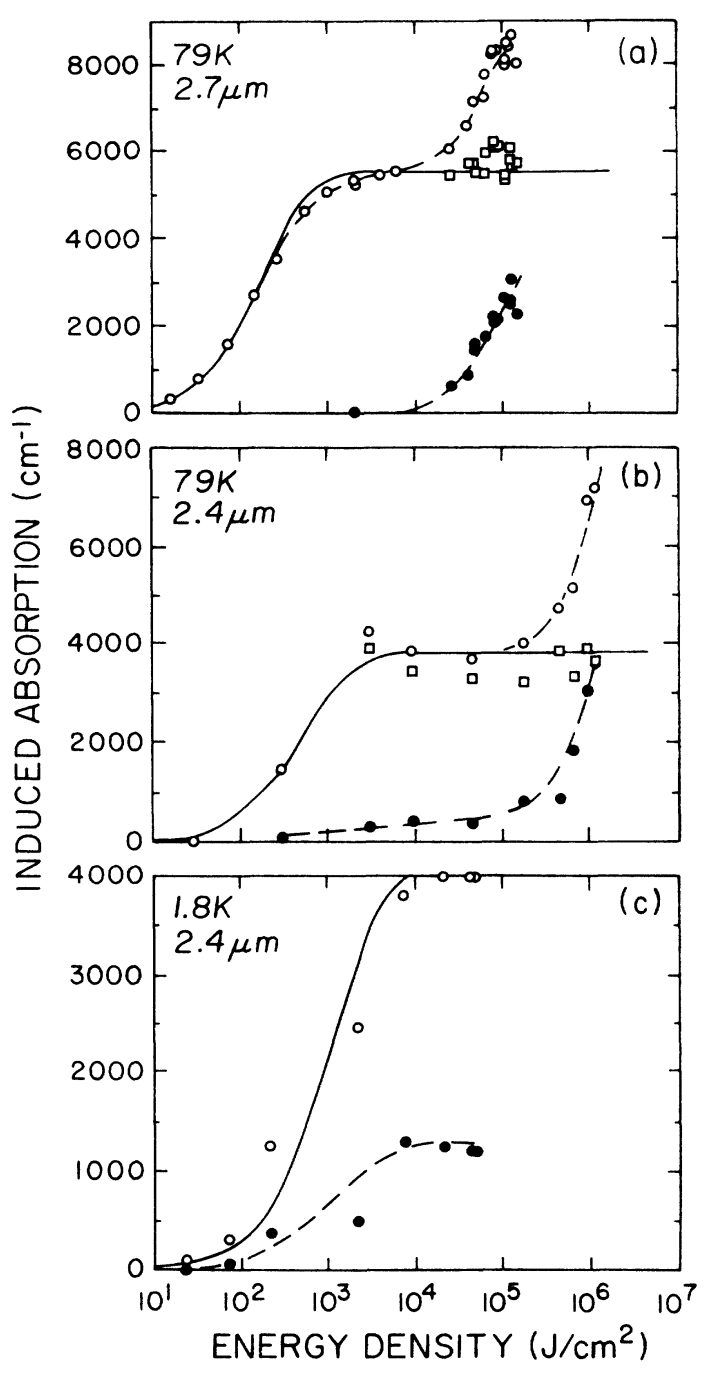

FIG. 8. Evolution of the average induced absorption (at 5145 $\AA$ ) (open circles), the midgap absorption measured at $\sim 6000 \AA$ (solid circles) and the difference (open squares, when different from open circles) which is associated with the photodarkening as explained in the text. The solid lines are the solutions of the direct process model [Eq. ( $\left.7^{\prime}\right)$ ] with $\alpha_{\infty}$ determined from the data and $\alpha_{0}=0$. (a) Thickness $2.7 \mu \mathrm{m}$ and intensity $7 \mathrm{~W} / \mathrm{cm}^{2}$ at $79 \mathrm{~K}$. (b) Thickness $2.4 \mu \mathrm{m}$ and intensity $3.8 \mathrm{~W} / \mathrm{cm}^{2}$ at $79 \mathrm{~K}$. (c) Thickness $2.4 \mu \mathrm{m}$ and intensity $8.8 \mathrm{~W} / \mathrm{cm}^{2}$ at $1.8 \mathrm{~K}$.

contribution from defects producing increased midgap absorption (solid circles, measured at $\sim 6000 \AA$ and assumed to be the same at $5145 \AA$ ) the portion we attribute to photodarkening (open squares where they differ from open circles) appears to saturate at approximately 5500 $\mathrm{cm}^{-1}$ after an exposure of approximately $2 \mathrm{~kJ} / \mathrm{cm}^{2}$ [solid line in Fig. 8(a)]. At low temperatures after optical excitation, midgap absorption ${ }^{9,12-14}$ appears which is essentially constant in magnitude from energies of about half the optical band gap up to energies where one can no longer measure this absorption due to the much greater background absorption of the exponential opticalabsorption edge (or perhaps also due to photodarkening).
We have approximated the contribution of this midgap absorption by measuring at $\sim 6000 \AA$, well below the band edge, and assuming an identical contribution at $5145 \AA$. The subtraction in Fig. 8(a) is consistent with this absorption. We provide further justification for this subtraction below.

The sample was further exposed from the back with 4 $\mathrm{kJ} / \mathrm{cm}^{2}$ producing a further increase in absorption at $5145 \AA$ of $334 \mathrm{~cm}^{-1}$. The small additional increase in absorption during this additional exposure verifies that the sample was essentially uniformly darkened. A second sample of $\mathrm{As}_{2} \mathrm{~S}_{3}$ which was $2.4 \mu \mathrm{m}$ thick was similarly exposed at $79 \mathrm{~K}$ at an intensity of $3.3 \mathrm{~W} / \mathrm{cm}^{2}$ for a total energy density of $1154 \mathrm{~kJ} / \mathrm{cm}^{2}$ [Fig. $8(\mathrm{~b})$ ] producing a total darkening of $7200 \mathrm{~cm}^{-1}$ at $5145 \AA$, but again, when the contribution from the absorption measured at $\sim 6000 \AA$ is subtracted the change in absorption due to photodarkening saturates at $\sim 4000 \mathrm{~cm}^{-1}$ after an exposure of approximately $3 \mathrm{~kJ} / \mathrm{cm}^{2}$. Notice that in this case there appear to be two measurable contributions to the absorption at $\sim 6000 \AA$, a small portion apparent at energy densities $\leq 10^{5} \mathrm{~J} / \mathrm{cm}^{2}$ which follows the photodarkening curve at $5145 \AA$, and a larger contribution at higherenergy densities $\left(\geq 10^{5} \mathrm{~J} / \mathrm{cm}^{2}\right)$ which is attributed to the energy-independent, midgap absorption. The ratio of the contributions at 5145 and $6000 \AA$ which we have attributed to photodarkening in Fig. $8(\mathrm{~b})$ is essentially the same as the initial ratio of the exponential Urbach edge absorption and is therefore consistent with the wellknown fact that photodarkening produces an essentially parallel shift of the absorption edge.

When another portion of the same $2.4-\mu \mathrm{m}$-thick sample (a previously unexposed region) was exposed at $1.8 \mathrm{~K}$ to an intensity of $8.8 \mathrm{~W} / \mathrm{cm}^{2}$, saturation was apparent after approximately $6 \mathrm{~kJ} / \mathrm{cm}^{2}$ exposure [Fig. 8(c)]. No evidence of a contribution from the energy-independent, midgap absorption (which is due to different defects as discussed below) appears up to the $46 \mathrm{~kJ} / \mathrm{cm}^{2}$ total exposure which is below the exposures which produced significant midgap absorption in Figs. 8(a) and 8(b) (50 and $200 \mathrm{~kJ} / \mathrm{cm}^{2}$, respectively). The absorption measured at $\sim 6000 \AA$ in Fig. 8(c) appears to be entirely due to photodarkening and scales approximately as one would expect from the initial absorption edge. The solid lines in Figs. 8(a)-8(c) are the solutions of Eq. $\left(7^{\prime}\right)$ after adjusting the length $\left(\alpha_{\infty}\right)^{-1}$ and time $(\gamma)^{-1}$ scales.

The induced absorption spectra shown in Fig. 9 demonstrate the evolution of the photodarkening and the midgap absorption during exposure. The photodarkening is characterized by a nearly uniform shift of the exponential band tail, ${ }^{7}$ hence the portions with constant slope at short wavelengths in Fig. 9. At higher exposure the midgap absorption appears as a uniform increase in absorption at all wavelengths from the gap to the near infrared. This absorption has been previously associated with light-induced electron spin resonance, ${ }^{8,9}$ (ESR) because of the following observation: (1) The midgap absorption and light-induced ESR occur without measurable photodarkening when below-gap light is used; ${ }^{12}$ (2) photodarkening is still readily observed (though smaller) after above-band-gap illumination at room temperature 


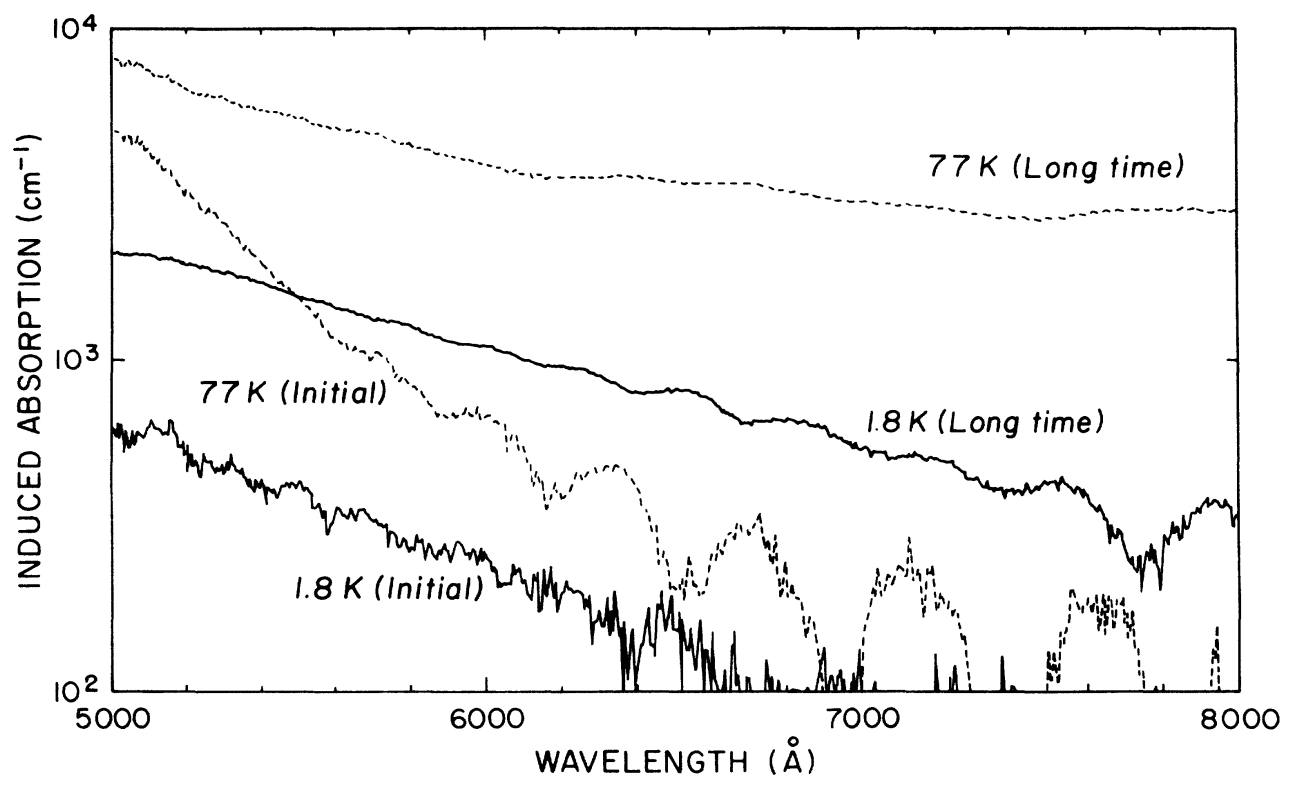

FIG. 9. Induced absorption $[\overline{\Delta \alpha}(\lambda)]$ spectra for the sample from Figs. 8(b) and 8(c) at $1.8 \mathrm{~K}$ (solid lines) and $77 \mathrm{~K}$ (dashed lines) after exposures shown.

while the induced midgap absorption has not been observed $\left(\Delta \alpha<1 \mathrm{~cm}^{-1}\right)$; (3) both the midgap absorption and the ESR signal induced by above-band-gap illumination at low-temperature anneal at lower temperatures $(\sim 170 \mathrm{~K})$ than does photodarkening $(>300 \mathrm{~K})$. Therefore, with the additional evidence provided by Fig. 9, we can assume that the midgap absorption is produced by a set of defects separate from those producing photodarkening. This provides additional evidence to support our justification for the subtraction of the midgap absorption in Figs. 8(a) and 8(b). The two sets of defects may not be truly independent as, for example, they may share an intermediate state. The temporal behavior of both the light-induced midgap absorption and light-induced ESR depend strongly on the intensity of the exciting light. Both of these defects appear to saturate after only $\sim 1$ $\mathrm{J} / \mathrm{cm}^{2}$ exposure at exciting light intensities below 10 $\mathrm{mw} / \mathrm{cm}^{2}$ while at higher intensities $\left(>100 \mathrm{~mW} / \mathrm{cm}^{2}\right)$ there is a dramatic increase in both the midgap absorption (see Fig. 8) and also in the induced ESR (Ref. 14) which continues to grow without apparent saturation for large exposures $\left(>100 \mathrm{~kJ} / \mathrm{cm}^{2}\right)$.

To determine further the actual profile of the photodarkening, we first photodarkened a $120-\mu \mathrm{m}$-thick wafer of $\mathrm{As}_{35} \mathrm{~S}_{65}$ glass at room temperature with $3.6 \mathrm{~W} / \mathrm{cm}^{2}$ for $5400 \mathrm{sec}$ and then polished the illuminated side, remeasured the transmission, polished again, etc., until the sample was $50 \mu \mathrm{m}$ thick. The result, reflecting the actual profile with a resolution of a few micrometers, is shown in Fig. 10. This experiment determined directly that the induced absorption is much larger in the first few micrometers of the sample surface and that the depth of photodarkening is less than $10 \mu \mathrm{m}$ at room temperature in this sample.

The photodarkening deep into a thick sample was profiled by the perpendicular illumination probe arrangement depicted in Fig. 6. This experiment measures the "tail" of the photodarkening profile from 1 to $6 \mathrm{~mm}$ into the sample where the photodarkening is less than 10 $\mathrm{cm}^{-1}$ even though the first few micrometers of the sample are darkened to several thousand $\mathrm{cm}^{-1}$. In this tail, the direct process profile Eq. (7) may be solved algebraically to yield

$$
\begin{aligned}
& \Delta \alpha \approx(\Delta \alpha)_{0}+A(t) e^{-\alpha_{\mathrm{tal}} x}, \\
& \alpha_{\text {tail }}=(\Delta \alpha)_{0}+\alpha_{0},
\end{aligned}
$$

where $(\Delta \alpha)_{0}=\left(\sigma_{d}-\sigma_{s}\right) N_{d}(t=0)$. The function $A(t)$ in-

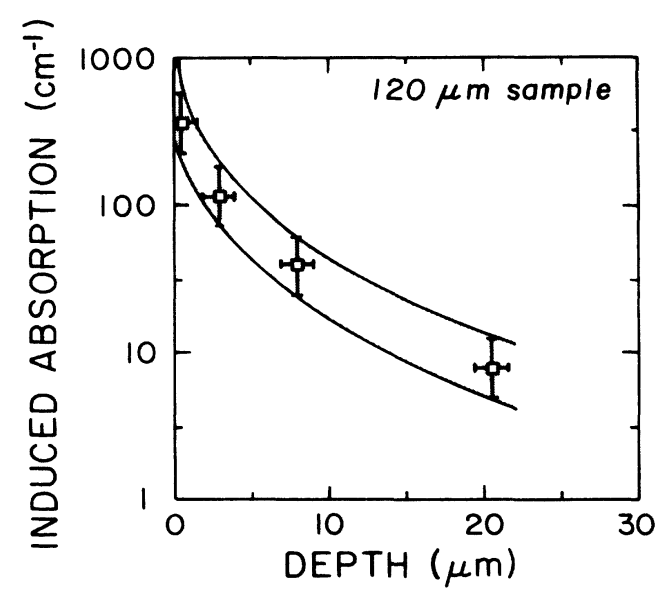

FIG. 10. The induced absorption profile, $\Delta \alpha(x)$ near the sur-

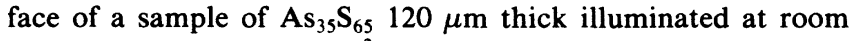
temperature by $3.6 \mathrm{~W} / \mathrm{cm}^{2}$ for $5400 \mathrm{sec}$. The inducing wavelength was $5145 \AA$ and the probe wavelength was $5500 \AA$. 


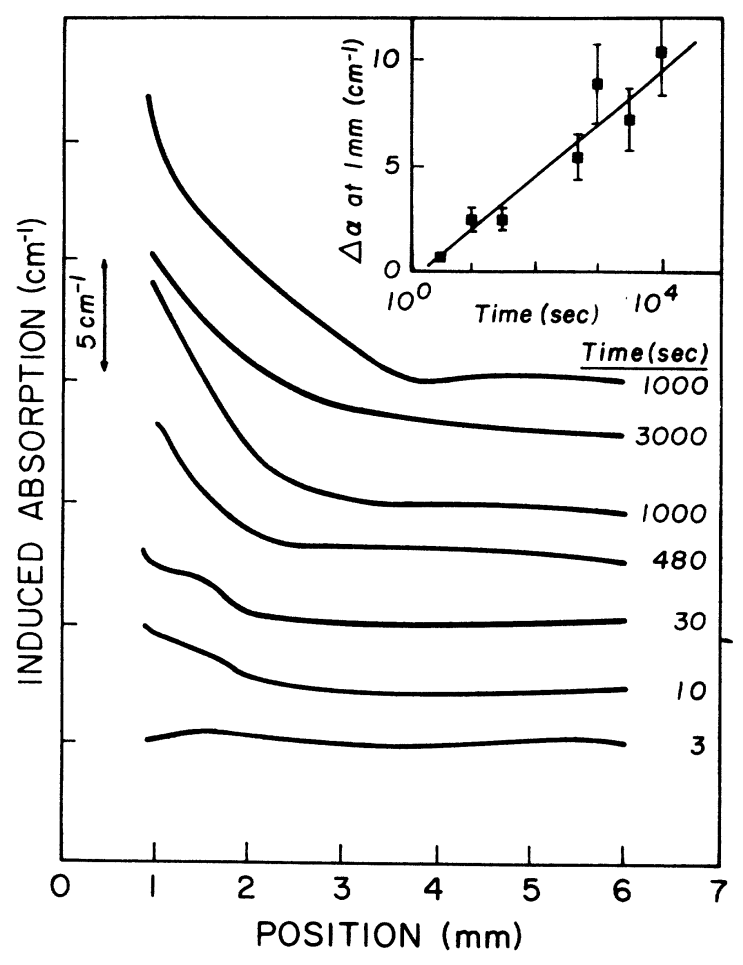

FIG. 11. Tail profiles of photodarkening deep (1-6 mm) into a thick $\mathrm{As}_{2} \mathrm{~S}_{3}$ sample using the perpendicular probe configuration of Fig. 7. The profiles at different times are displaced vertically for the clarity and the double arrow shows an absorption change of $5 \mathrm{~cm}^{-1}$. The inducing intensity was 1.8 $\mathrm{W} / \mathrm{cm}^{2}$ at $1.8 \mathrm{~K}$. The inset shows the evolution of the parameter $A(t)$ [see Eq. (12)] where the line is drawn as an aid to the eye.

creases from zero monotonically as $t$ increases and is approximately proportional to $\ln t$ for thick samples $\alpha_{\infty} L \gg 1$ [see Fig. 3(a)]. Figure 11 shows the tail profiles measured at $5300 \AA$ with 5145 - $\AA$ excitation at various times. (The absorption measured at $5300 \AA$ will likely include a contribution from the midgap absorption but it is still a measure of the total exposure at the different depths.) For all curves $\alpha_{\text {tail }} \approx 4 \mathrm{~cm}^{-1} \approx \Delta \alpha_{0}+\alpha_{0}$ at all times and is justifiably neglected compared to $\alpha_{\infty}>10^{4}$ $\mathrm{cm}^{-1}$. The inset shows the time evolution of $\Delta \alpha(x=1$ $\mathrm{mm})$, which is proportional to $A(t)$.

\section{CONCLUSIONS}

The measured time evolution of photodarkening in glassy $\mathrm{As}_{2} \mathrm{~S}_{3}$ is quantitatively consistent with a model which assumes that the rate of creation of photodarkened defects at any region in the glass is proportional to the photons absorbed in that region up to some maximum allowable defect density. The experimental data do not appear to be consistent with a model in which the creation of new photodarkened defects is proportional to the absorption of photons at existing photodarkened defects. The model, which has only two adjustable parameters, a saturated absorption length scale $1 / \alpha_{\infty}$ and a normalized time scale $1 / \gamma$, is also consistent with two depth profiling experiments. In the first experiment a photodarkened sample was progressively etched back from the surface at which the photodarkening light was incident, and the change in the measured (average) absorption coefficient was monitored as a function of etching depth. This experiment probed distances on a scale of 10 to $100 \mu \mathrm{m}$. In the second experiment the profiling was performed by measuring the transmission through a thin $(\sim 100 \mu \mathrm{m})$ sample after exposure to photodarkening light from one edge. This experiment probed lengths on a scale of 0.1 to $1 \mathrm{~cm}$. Photodarkening experiments on samples whose thicknesses are comparable to the saturated absorption length $\left(1 / \alpha_{\infty} \simeq 2.5 \mu \mathrm{m}\right)$ appear to demonstrate saturation of the photodarkening.

In some cases the interpretation depended upon subtraction of a frequency-independent below-gap absorption from the photodarkening, which is rapidly varying with frequency. This subtraction is substantiated by independent experiments ${ }^{8,9,12-14,19}$ which show that the optically induced midgap absorption and ESR result in a different set of defects from those producing the photodarkening.

Although these experiments represent the first demonstration of a saturation of the photodarkening effect in bulk samples, saturation has been observed previously on thin films of glassy $\mathrm{As}_{2} \mathrm{~S}_{3}$ made by evaporation or sputtering. ${ }^{7,16,18,20}$ In these earlier experiments the values of $1 / \alpha_{\infty}$ ranged from 1500 to $5000 \mathrm{~cm}^{-1}$ at $2.41 \mathrm{eV}$ in good agreement with the values reported here in bulk, meltquenched glasses. Because the structures of films of $\mathrm{As}_{2} \mathrm{~S}_{3}$ are known to be different from the bulk glass, even when the films have been annealed, ${ }^{21}$ one should not $a$ priori expect the saturated values of the photodarkening to be exactly the same in these two forms of $\mathrm{As}_{2} \mathrm{~S}_{3}$ glass.

One may ask what causes saturation of the photodarkening process. The most obvious, but perhaps least enlightening, answer is that there exist only a finite number of photodarkening sites which can presumably be no greater than the number of atoms (or perhaps the number of bonds) in the glass. It is difficult to estimate the total integrated absorption produced by the photodarkening or the matrix elements which are involved in these optical transitions, but reasonable upper bounds suggest that the saturated value of the photodarkening $\left(4000-5000 \mathrm{~cm}^{-1}\right.$ at $2.41 \mathrm{eV}$ ) corresponds to at most $1 \%$ of the available sites in the glass. This number is consistent with $\mathrm{x}$-ray scattering and extended $\mathrm{x}$-ray-absorption fine-structure (EXAFS) experiments ${ }^{2-24}$ on $\mathrm{As}_{2} \mathrm{~S}_{3}$ films before and after photodarkening which indicate that less than $1 \%$ of the bonds between nearest neighbors have been altered.

This value of $1 \%$ of the available atomic sites could result from several causes. There could exist a maximum number of preexisting, localized defects in the glass which are predisposed to become photodarkened sites. A second possibility is that the defects have a more extended range such that at $1 \%$ the interactions between photodarkened defects limit any further net creation of defects. If the conversion of an unphotodarkened site to a photodarkened site involves rearrangements or distortions over several atomic sites, then further conversion may be hin- 
dered when the sites begin to overlap. Such overlap will occur at a density which is approximately $1 /$ (characteristic length $)^{3}$ of the total atomic density. Recent $x$-ray scattering experiments ${ }^{23}$ have observed longer-range (second nearest neighbor) distortions in the glassy network after photodarkening in thin films of $\mathrm{As}_{2} \mathrm{~S}_{3}$ in agreement with the assumption that there is large overlap of photodarkened sites.

A third possibility is that the probability that the absorbed photon will bleach a photodarkened site equals the probability for creation of a site at the value of $1 \%$. It is well known ${ }^{7,13,18}$ that below-gap light, which does not efficiently create photodarkening, is effective in bleaching the photodarkening centers. There is no reason to expect that above-band-gap light will not also bleach these centers. The second terms in Eqs. (1) and (8) are the bleaching terms and their effect on $\alpha_{\infty}$ can be clearly seen in Eqs. (5) and (9), respectively. When $\sigma_{d} \phi_{d}$ is comparable to $\sigma_{s} \phi_{s}$, then $N_{d}<N_{s}$ [because $\alpha_{\infty}=\left(\sigma_{d}-\sigma_{s}\right) N_{d}<\sigma_{d} N_{s}$ and $\left.\sigma_{s} \ll \sigma_{d}\right]$.

At the high light intensities and at the probing energies which we have used, there is a relatively frequency in- dependent midgap absorption which continues to grow with optical excitation after the photodarkening has saturated. The values of this midgap absorption can exceed those of photodarkening at $2.41 \mathrm{eV}$ in glassy $\mathrm{As}_{2} \mathrm{~S}_{3}$. We have justified our separation of these two effects based on the spectral dependence of the absorption, but clearly at the highest densities [cf. Fig. 8(b)] these two contributions may no longer be independent. However, the model is still consistent at times greater than the saturation of the photodarkening but less than the rise of the midgap absorption to comparable values.

\section{ACKNOWLEDGMENTS}

The authors were aided by discussions with J.Z. Liu in the course of this work and by technical assistance from J. Viner and B. Moosman. This work was supported by the National Science Foundation under Grant No. DMR-86-15217 and by the Office of Naval Research, U.S. Department of Defense, under Grant No. N0001490-J-1222.
*Present address: IBM Research Division, Almaden Research Center, 650 Harry Road, San Jose, CA 95120-6099.

${ }^{1}$ J. P. DeNeufville, S. C. Moss, and S. R. Ovshinsky, J. NonCryst. Solids 13, 191 (1973/1974).

2J. S. Berkes, S. W. Ing, Jr., and W. J. Hillegas, J. Appl. Phys. 42, 4908 (1971).

${ }^{3}$ K. S. Harshavardhan and M. S. Hegde, Phys. Rev. Lett. 58, 567 (1987).

${ }^{4}$ Ke. Tanaka and A. Odajima, Solid State Commun. 12, 961 (1982).

${ }^{5}$ U. Strom, W. M. Pontusuhka, D. J. Treacy, and P. C. Taylor, J. Phys. Soc. Jpn. Suppl. A 49, 1155 (1980).

${ }^{6}$ E. Mytilineou, P. C. Taylor, and E. A. Davis, Solid State Commun. 35, 497 (1980).

${ }^{7}$ Ke. Tanaka, Jpn. J. Appl. Phys. 25, 779 (1986).

${ }^{8}$ S. G. Bishop, U. Strom, and P. C. Taylor, Phys. Rev. Lett. 34, 1346 (1975).

${ }^{9}$ S. G. Bishop, U. Strom, and P. C. Taylor, Phys. Rev. B 15, 2278 (1977).

${ }^{10}$ H. Hamanaka, K. Tanaka, A. Matsuda, S. Iizima, Solid State Commun. 19, 499 (1976); K. Kimura, H. Nakata, K. Murayama, and T. Ninomiya, ibid. 40, 551 (1981).

${ }^{11}$ J. Cernogora, F. Mollot, and C. Benoit á la Guillaume, Phys. Status Solidi A 21, 281 (1974); B. T. Kolomiets, T. N. Mamontova, and V. A. Vassilyev, in Proceedings of the 7th International Conference on Amorphous and Liquid Semiconductors, edited by W. E. Spear (University of Edinburgh, Edinburgh, 1977), p. 627.

${ }^{12}$ J. A. Freitas, Jr., U. Strom, and S. G. Bishop, Phys. Rev. B 35, 7780 (1987).
${ }^{13}$ J. Hautala, W. D. Ohlsen, and P. C. Taylor, Phys. Rev. B 38, 11048 (1988).

14J. Hautala, S. Ducharme, and P. C. Taylor, in The Physics of Semiconductors, edited by W. Zawadzki (Polish Academy of Sciences, Warsaw, 1988), Vol. II, p. 1677.

15J. Z. Liu and P. C. Taylor, Phys. Rev. Lett. 59, 1938 (1987).

${ }^{16} \mathrm{~K}$. Tanaka, in Fundamental Physics of Amorphous Semiconductors, edited by F. Yonezawa (Springer-Verlag, New York, 1981), p. 104.

${ }^{17} \mathrm{~S}$. J. Hudgens and M. Kastner, in Proceedings of the 7th International Conference on Amorphous and Liquid Semiconductors, edited by W. E. Spear (University of Edinburgh, Edinburgh, 1977) p. 622.

${ }^{18} \mathrm{Ke}$. Tanaka and Y. Ohtsuka, Thin Solid Films 33, 309 (1976).

${ }^{19}$ J. Z. Liu and P. C. Taylor, in Amorphous and Liquid Semiconductors, edited by F. Evangelisti and J. Stuke (North-Holland, Amsterdam, 1985), p. 543; P. C. Taylor and J. Z. Liu, in Defects in Glasses, edited by F. L. Galeener, D. L. Griscom, and M. Weber (Materials Research Society, Pittsburgh, 1986), Vol. 61, p. 223.

${ }^{20} \mathrm{~K}$. Tanaka, Appl. Phys. Lett. 26, 243 (1975).

${ }^{21}$ D. J. Treacy, U. Strom, P. B. Klein, P. C. Taylor, and T. P. Martin, J. Non-Cryst. Solids 35+36, 1035 (1980).

${ }^{22}$ C. Y. Yang, J. M. Lee, M. A. Paesler, and D. E. Sayers, J. Phys. (Paris) Colloq. 47, C8, 387 (1986); C. Y. Yang, M. A. Paesler, and D. E. Sayers, Phys. Rev. B 36, 9160 (1987).

${ }^{23}$ G. Pfeiffer, C. J. Brabec, S. R. Jefferys, and M. A. Paesler, Phys. Rev. B 39, 12861 (1989).

${ }^{24}$ C. Y. Yang, M. A. Paesler, and D. E. Sayers, Phys. Rev. B 39, 10342 (1989). 


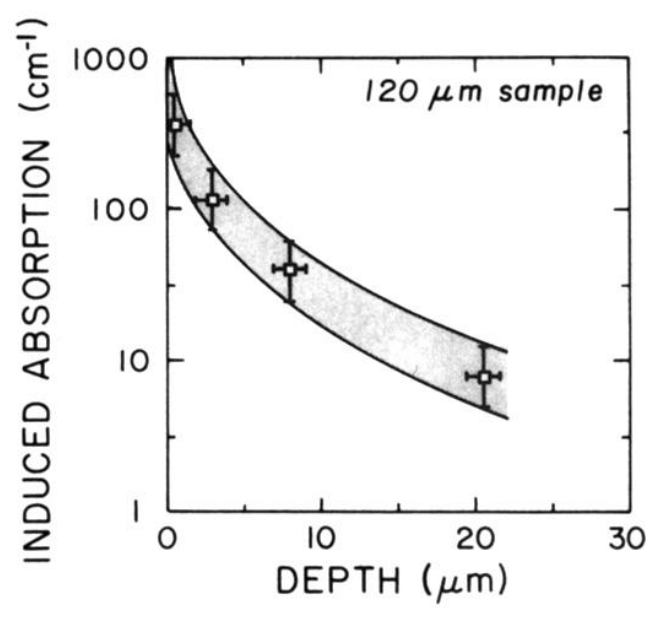

FIG. 10. The induced absorption profile, $\Delta \alpha(x)$ near the surface of a sample of $\mathrm{As}_{35} \mathrm{~S}_{65} 120 \mu \mathrm{m}$ thick illuminated at room temperature by $3.6 \mathrm{~W} / \mathrm{cm}^{2}$ for $5400 \mathrm{sec}$. The inducing wavelength was $5145 \AA$ and the probe wavelength was $5500 \AA$. 